\title{
Deposition of ordered arrays of copper nano dots by using self- assembled nanoporous silica as the template
}

\author{
Hufang $\mathrm{Xu}^{*}$, Ying-Bing Jiang*, Nanguo Liu** and Yifeng Wang ${ }^{* * *}$ \\ * TEM lab, Department of Earth and Planetary Sciences, University of New Mexico \\ ** Department of Chemical and Nuclear Engineering, University of New Mexico \\ *** Sandia National Laboratories, Carlsbed, NM88220
}

Because of their small size and large surface area, nano particles have been known to posses some unusual optical, electrical and magnetic properties that can not be found from its bulk states. Some of those properties, like the bandgap of some semiconductor nano dots, are tunable by adjusting the particle size and the particle arrangement. So, it is of particular interest to get ordered arrays of nano particles with uniform particle size.

In our experiment, using a self-assembled porous silica film as the substrate, ordered arrays of copper nano dots have been made by ion beam sputtering deposition. The selfassembled nanoporous silica film was made by spin coating with a kind of silica sol-gel solution. There are some P123 surfactant in this silica sol-gel solution, which will form ordered arrays of micelles when the gel solidifies and leave ordered arrays of nano pores after heat treatment at $450{ }^{\circ} \mathrm{C}$. The copper deposition was performed in an typical ion beam sputtering deposition system. The beam energy was $4.5 \mathrm{keV}$ and the beam current was $1.2 \mathrm{~mA}$. Beside the $\mathrm{Ar}^{+}$beam for sputtering deposition, an additional $\mathrm{Ar}^{+}$beam was used to enhance the copper deposition inside the nanopores as well as to clean up the copper deposition on the top surface of the sample. JEOL $2010 \mathrm{~F}$ transmission electron microscope (TEM) was used to characterize the sample. Selected area electron diffraction and high-resolution images have been obtained from the copper dots.

Figure 1 is the TEM results for our sample. Figure 1A is the TEM image of the nanoporous silica deposited with copper. Many ordered arrays of dark dots can be seen from the silica matrix. The size of those dots is uniform at around $5 \mathrm{~nm}$. Figure 1B is the Fourier form transformation (FFT) pattern for the area shown in Figure 1A, indicating a periodical arrangement of the nano dots. Figure $1 \mathrm{C}$ is the electron diffraction pattern from the same area. These rings in this can be indexed as copper 111, 200, and 220, respectively. Figure 1D is the image of nanoporous silica without copper deposition. Figure 1E is the high resolution TEM image for those copper dots. Lattice fringes can be seen from this image. And the FFT pattern (Figure 1F) for the fringes are in agreement with copper lattice. Both the diffraction pattern in Figure 1C and the FFT pattern in Figure $1 \mathrm{~F}$ demonstrate that the dark dot in figure $1 \mathrm{~A}$ are copper nano dots.

This research is supported by NSF(EAR02-1082). Huifang Xu: hfxu@unm.edu 

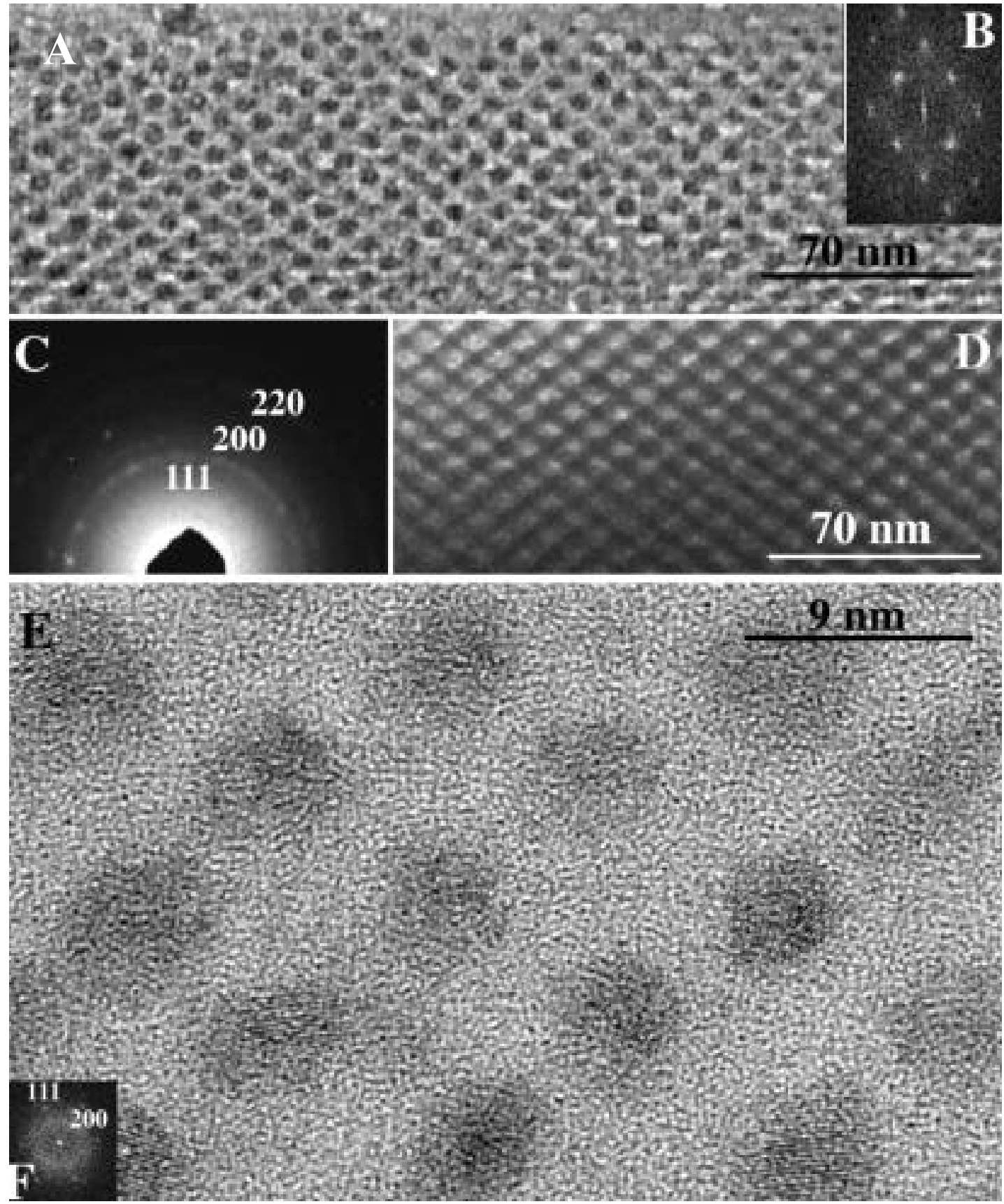

Figure 1. TEM images for nanoporous silica embedded with copper nano dots. 